\title{
Practical Assessment of MRI Coil's Characteristics
}

\author{
Dia M. Ali \\ University of Ninevah \\ Mosul / Iraq
}

\begin{abstract}
Magnetic Resonance coil is one of the limitation and degradation of image quality in Medical Imaging. Ghosting, Artifacts, and noise are the main consequence of the mismatch of the receiving coil. Human body parts have deferent permittivity and permeability which cause a variation of the self-resonance inductance.

I this paper, the effects of variable material filled in brain coil is studied and measured. The different arrangement also performed. The results show a noticeable variation of the selfresonance inductance which yields drifting the operating frequency by $122 \mathrm{KHz}$ which is beyond the bandwidth of the MRI signal and instrument. One of the solutions is the autotuning algorithm, which is the only available solution. As a proposed solution, a cylindrical slab with high relative permittivity can be used inside the coil winding which reduce the variation of the operating frequency..
\end{abstract}

\section{General Terms}

Nuclear magnetic resonance. Coil,

\section{Keywords}

MRI, Receiving Coils, Q Point..

\section{INTRODUCTION}

Magnetic resonance imaging (MRI) became the most useful tool in the diagnosis and patient healthcare. The main reason for deploying of MRI is safety, where no ionizing radiation is used compared with other imaging techniques. All other equipment based on the ionizing radiation such as Computed tomography (CT), X-rays, and positron emission tomography (PET) can be harmful. Nuclear magnetic resonance (NMR) phenomenon, which the fundamental of MRI does not cause prejudicial effects on the patient's health.

MRI can be classified based on the main magnetic field (B0), Open type and Closed close type. The field strength (B0) of the close type Time go from 1.5 to 3 Tesla and even to 7 Tesla. As the Field B0 increases, the advantages of better signal to noise ratio (SNR), spatial resolution, and sensitivity among others also increases[1].

Open time, on the other hand, has many advantages over the close time, such as patient comfort, increasing the scan volume, lower maintenance cost, and other logistic problems.

The main components of the MRI are shown in Figure (1)[2]

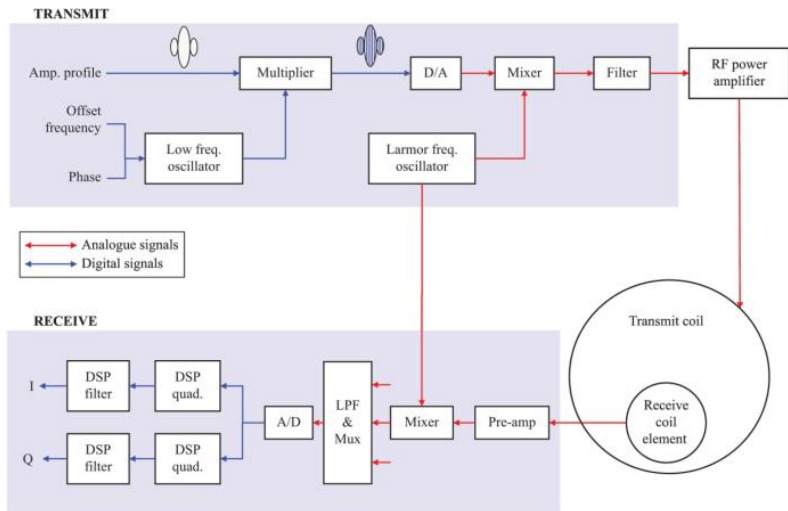

Fig 1: Main MRI Component

The main principle of Magnetic resonance imaging is the "nuclear magnetic resonance (NMR)", which is concerned with the spin property of protons in the human body. These phenomena can be represented as a ball rotating about its axis, which yields an angular and a magnetic momentum $\mu$. The energy can be stored in this spinning protons using a pulse of a higher energy radio frequency (RF) ( also called B1). This saved energy is released after a period depending on the types of the tissue. Spatial encoding is used to precisely measure the saved energy, and with the aid of construction algorithms, a 3D image can be achieved. [2]

The excitation of the B1 needs antenna works within its frequency known as Coil. The operating frequency covered by the field B0 and can be calculated as

$\mathrm{f}_{\text {Larmor }}=\bar{\gamma} \mathrm{B} 0$

where $\mathrm{f}$ is the Larmor frequency in $\mathrm{Hz}$

$$
\bar{\gamma} \text { is the gyromagnetic ratio }
$$

\section{and $\quad$ B0 is main magnetic field strength}

The frequency of operation produces RF problems (Larmor frequency), where some parts are in deep location, and the penetration depth is proportional to the RF power, ie $\mathrm{B}+1$ field. It is obvious that increasing the excitation power will increase the energy deposition which sometimes beyond specific absorption rate limitations, leading safety issues due to local overheating of the human body parts. Many researchers proposed a variety of solutions to overcome the challenge of generating a penetrating and homogeneous excitation RF field (B1) inside the body part while keeping low levels of energy deposition [2]

There are different methods to solve these RF related problems such as RF multi-transmit techniques and coil array design [3].

This paper on the letter focused on RF coil parameters and limitation in open time MRI especially $0.23 \mathrm{~T}$. It's obviously that better Coils gains and impedance matching results better performance and better Signal to Noise ratio and hence minimizing the excitation $\mathrm{Rf}$ power than reducing the SAR 
The body effects on the characteristic behavior of the receiver coil and cause a severe mismatch, and consequently, a continues calibration and signal optimization is monolatry required.

\section{RECEIVING COIL MODEL}

In MRI, generally, every part of the human body has its own Receiving coil type and configuration. Some types called flexible, others use taped configuration, and most of them use dual channel configuration. In this paper, dual channel Brian coil is utilized.

There are four general designs, multiple turn solenoid, single turn surface coil, birdcage coil and saddle coil. All produce the B 1 field in the direction shown, and all but the solenoid are used readily in horizontal bore scanners as shown in Figure (2)

The RF coil should be of similar size to the anatomy of interest to maintain good SNR, as the signal comes from the imaged slice but the noise is from the entire sensitive volume of the coil.

The Receiving coil can be classified int to groups according to their configuration. The first group is the volumetric type where the coil surrounds the body part from all direction. This types also used for transmitting and receiving signals due to the uniform field in a large surrounding volume. Helmholtz coil, as an example, is consist of couple of two identical circular loops, the separation distance equal to the loop radius to ensure a uniform field generation in the vicinity of their midpoint as shown in figure ( 2 ) right[5]

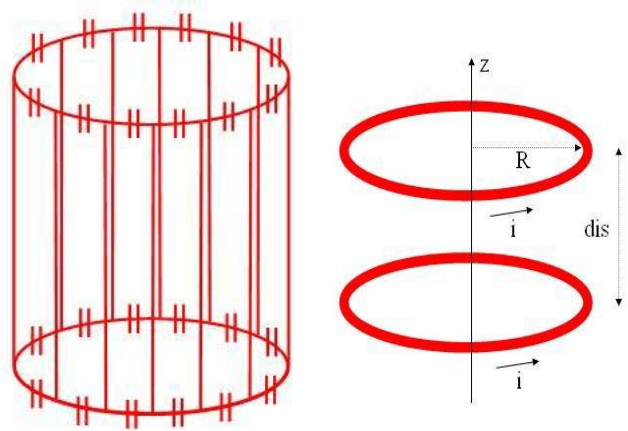

Fig. 2 Helmholtz coil (right ) and birdcage coil (left)

Birdcage coil, on the other hand, is favorite because it has the ability to generate a highly homogenous RF magnetic field with relatively high signal-to-noise ratio (SNR), and from its name, its look like a birdcage with $\mathrm{N}$ legs connected with two circular loops at each end Fig. 1 Left ) [6].

The second group contained surface coils, and it consists of a closed loop with various shapes and its out of our scope.

Array coils ( Quadrature coil as an example) provide a large region of sensitivity, similar to that obtained with volume coils, and a high SNR. These array elements, constituted by circular or rectangular loops, and each loop is connected to a receiver channel independently. Phase correction and channel equalization is applied to enhance the overall Signal to noise ratio [7]

One of the methods used to achieved coupling between coil elements is by overlapping the adjacent coils to minimizing mutual inductance (or eliminate it). Using low input impedance preamplifier, on the other hand, is used to reduce the interference between the overlapped couple and the other coils in the array[8].
The basic model for MRI coil is the simple RLC model as shown in figure (2). It's obvious, by reciprocal principle, this coil be either transmit or receive coil.

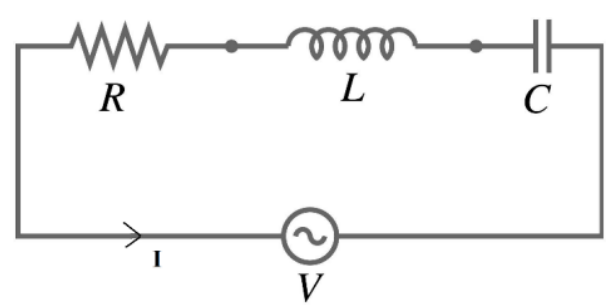

Fig. 3 Self Resonance coil model

Where $\mathrm{L}$ is the system inductance and $\mathrm{C}$ is the system capacitance and they are mainly related to the conductor size and geometry. The resistor R, on the other hand, is the combination of Rcoil takes into account for the losses within the coil conductors and depends on the conductor geometry and Rsample are the sample losses caused by RF currents and Rextra include radiative and tuning capacitors losses, which can be neglected in many applications.

The resonant frequency $\omega$ of the coil is related to both its inductance $\mathrm{L}$ and its capacitance $\mathrm{C}$ by:

$\omega=1 / \sqrt{\mathrm{LC}} \quad \ldots . . . .(3)$

and the quality factor $\mathrm{Q}$ can be calculated as: $Q=\omega L /_{R} \ldots \ldots \ldots \ldots . .(4)$

\section{FILLED COIL CHARACTERISTICS}

Self-resonance (SRF) coil can be modeled using the transmission line model, where the effective dielectric constant near to the surface of the former needs to be considered because this is the region has most of the field is concentrated. Using Lorentz local field formula, an effect of the dielectric constant calculated. Note that this formula is applied to cylinders neglecting edge effects or spheres. So for the transmission line model used as the effective dielectric constant, this is given by:

$$
\bar{\varepsilon}=1+\frac{\eta}{\frac{1}{\epsilon_{1}-1}+\frac{1}{p}(1-\eta)} \quad \ldots \ldots .2
$$

Where $\eta$

is the volume fraction $=4 \mathrm{da} / \mathrm{D}$ for infinitely long cylinders where $\mathrm{D}$ is the diameter of the former, da is the cylinder thickness.

Here $\mathrm{P}=2$ for cylinders

$\epsilon_{1}$ is the dielectric constant of the cylinder.

The extended form of the above equation can be written as :

$\overline{\bar{\varepsilon}}=1+\frac{\ddot{\bar{\epsilon}}-1}{\bar{\epsilon}+(p-1)}\left(\frac{D+d_{w}}{D}\right)^{p} \quad \ldots . .3$

where: $\mathrm{dw}$ is the wire thickness

The operating frequency of such coil is governed by Equation (1), since the Bo is $0.23 \mathrm{~T}$ the operating frequency yields $9.8 \mathrm{MHz}$. Figure (2) illustrates the basic configuration of Brain coil. Avery efficient RF coil design utilizing many regularly spaced conductors giving its characteristic appearance and name (Figure 24). Also referred to as a distributed capacitance coil. Most head coils are of this design, typically used as a transceiver produces very homogeneous imaging profiles, particularly in the axial plane. [4 ]

\section{PRACTICAL RESULTS}

The measurement device used was 4192A Impedance 
Analyzer. The coil was a two-channel brain coil as shown in Figure (4)

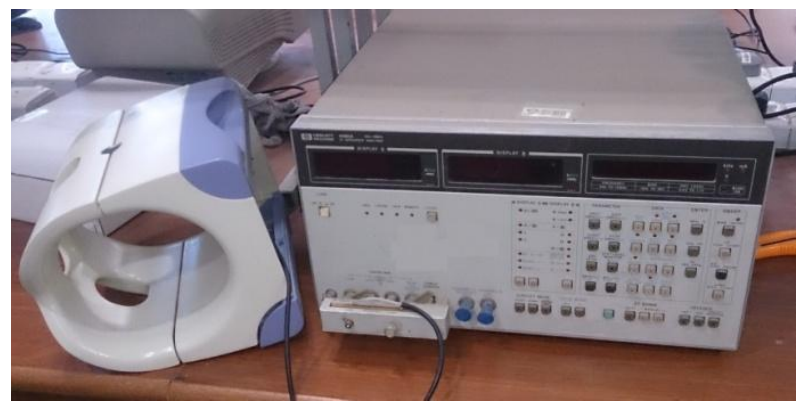

Fig 4: Measurement Setup

The coil connected with $50 \mathrm{Ohm}$ cable to the impedance analyzer and its length is neglectable with respect rot the operating wavelength as shown in Figure (5)

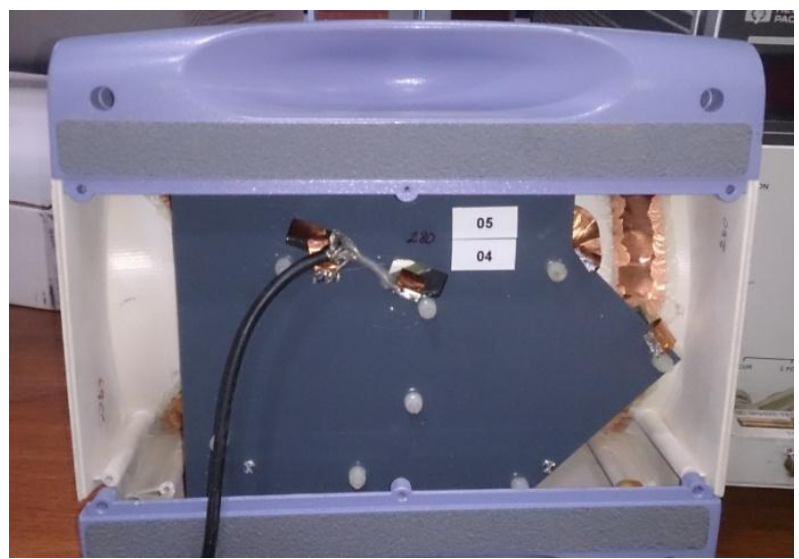

Fig 5: Coil Connection

\section{1}

In this paper, the brain coil shown in the figure(5 ) is used to study its behavior in the deferent environment. As a first step, the effect of the frequency on the inductance value $L$ and resistivity R. Figure ( 6 ) show results obtained, where both $\mathrm{L}$ and $\mathrm{R}$ seems to increase with the frequency.

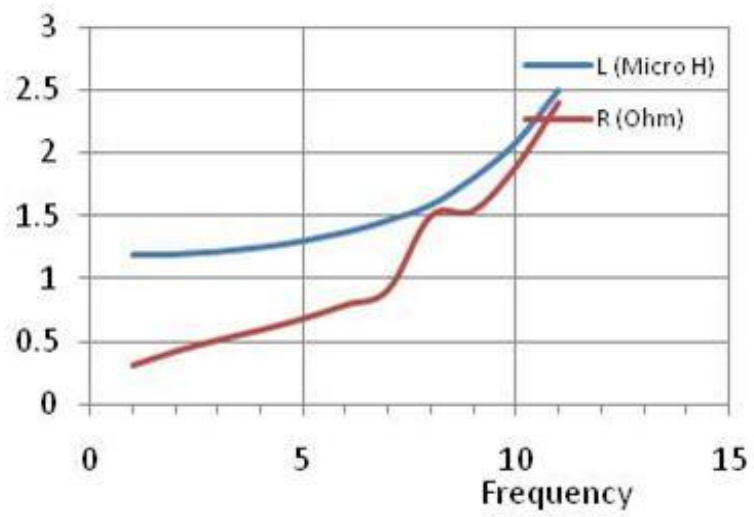

Fig 6: Air filled Coil characteristics

The second test was to study in deep and precisely on the operating frequency of the coil, ie $9.800 \mathrm{MHz}$. Note the that the required operating is within $20 \mathrm{KHz}$. The results show that the variation of $\mathrm{L}$ and $\mathrm{R}$ are $0.3 \%$ for $\mathrm{L}$ and less than 0.01 $\%$ for R.

Another test ( which is the most important) was to study of filling the coil with human body extremity. Of course, it is better to simulate the human body part with desterilized water(phantom) . Figure (7 ) shows the response of the coil, and it was evident that the variation of the inductance is within $0.3 \%$ and there is no noticeable variation in $\mathrm{R}$.

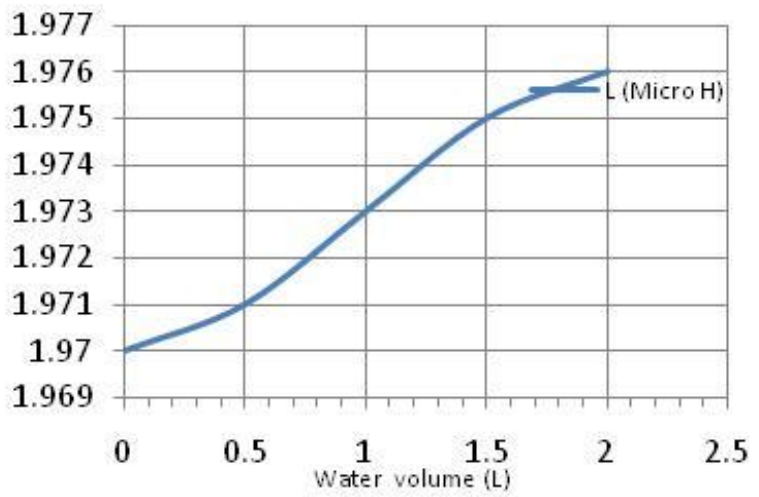

Fig 7: Water filled (phantom) Coil characteristics

\subsection{Extended Body effect}

In order to simulate the exact boundaries of the real operation of the MRI coil, Real Extremity ( Knee ) was used. The distance of the filled object in the coil was tested in the range $5-20 \mathrm{~cm}$, where the width of the coil also $20 \mathrm{~cm}$. Figure (8) shows the results) at the center frequency $(9.8 \mathrm{MHz})$.

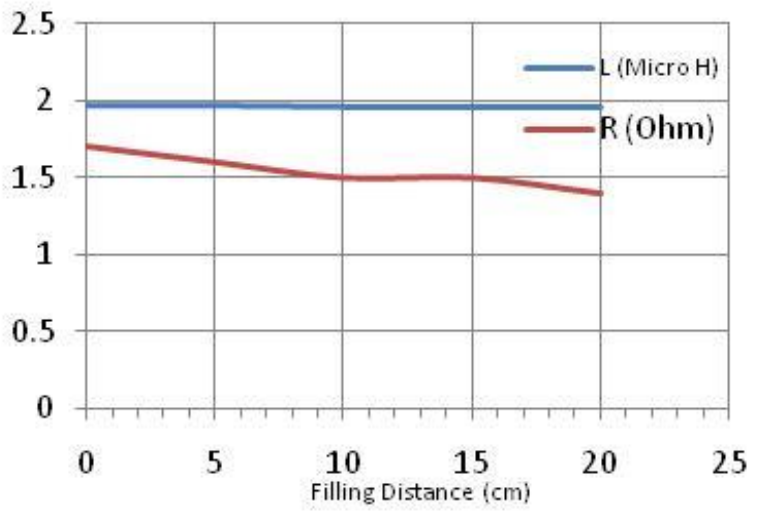

Fig 8: Water filled Coil characteristics at deferent

For comparison, three cases of filing is achieved, Air filled, 2 Litter Water filled, and Knee join filed. The results are shown in the Figure ( 9 )

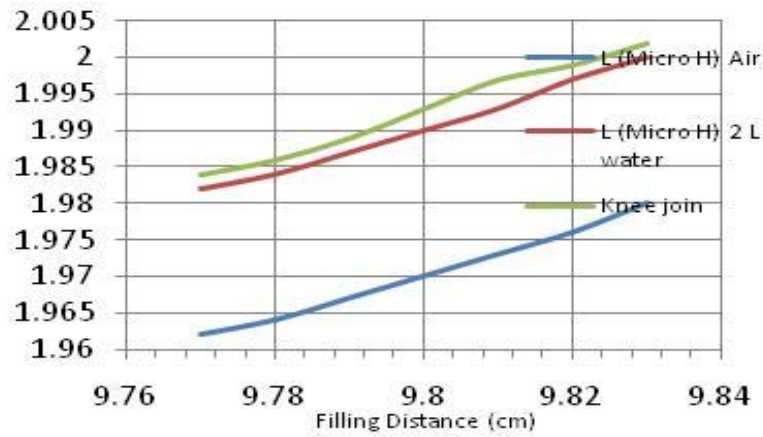

Fig 9: Coil characteristics at deferent filling objects

Note that the $\mathrm{Q}$ factor measured for the above arrangement was between $62-78$

\section{PROPOSED APPROACH}


The consequences of entering a human body part can be minimized Either by minimizing the effect of the increasing or decreasing the relative permittivity and relative permeability. Equation 2 summarized that when using a cylindrical slap with very high relative permittivity, the overall permittivity governed mainly by its permittivity.

Of course, this needs a super dielectric [9], with relative dielectric constant within the range of 109 [10 ]

\section{CONCLUSION AND SUGGESTION.}

It can be concluded from figures ( $6-9$ ) that the variation of the inductance for difference configuration can reach 1.25 $\%$ and this value is not small enough to be neglected since the $\mathrm{Q}$ factor for such coils is very high. Such variation yield to change the center frequency by at least $122 \mathrm{KHz}$ and the coil will be out of tuning. To overcome such problem, auto-tuning schedule required for each test[ references].

Mismatch problem due to human body effects can be minimized using a new cylindrical slab with high relative permittivity. The calculated results show that the frequency drift of the receiver coil due the human body is reduced by a ratio of the dielectric cylinder to the whole diameter and that's yield to minimize and simplified the recalibration process at the beginning of the scan.

\section{REFERENCES}

[1] J. Vaughan, M. Garwood, et. al "7T vs. 4T: RF power, homogeneity, and signal-to-noise comparison in head images," Magnetic Resonance in Medicine, vol. 46, no. 1, pp. 24-30, 2001.

[2] JJ. T. Vaughan, C. J. Snyder, L. J. DelaBarre, P. J. Bolan, J. Tian, L. Bolinger, G. Adriany, P. Andersen, J. Strupp, and K. Ugurbil, "Whole-body imaging at 7T: Preliminary results," Magnetic Resonance in Medicine, vol. 61, no. 1, pp. 244-248, $2009 .$.
[3] A. J. E. Raaijmakers, O. Ipek, D. W. J. Klomp, C. Possanzini, P. R. Harvey, J. J. W. Lagendijk, and C. a. T. van den Berg, "Design of a radiative surface coil array element at 7T: The single-side adapted dipole antenna," Magnetic Resonance in Medicine, vol. 66, no. 5, pp. 1488-1497, 2011.

[4] C. E. Hayes, W. A. Edelstein, J. F. Schenck, O. M. Mueller \&M. Eash (1985). An efficient, highly homogeneous radio frequency coil for whole-body NMR imaging at 1.5 T. J. Magn. Res., 63, 622-8.

[5] E.M. Haacke, R.W. Brown, M.R. Thompson, R. Venkatesan, Magnetic Resonance Imaging: Physical Principles and Sequence Design Book, Wiley-Liss, 1999.

[6] C.E. Hayes, W.A. Edelstein, J.F. Schenck, O.M. Mueller, M. Eash, "An efficient, highly homogeneous radio frequency coil for whole-body NMR imaging at 1.5 T", J. Magn. Reson., vol. 63(3), pp. 622-628, 1985.

[7] P.B. Roemer, W.A. Edelstein, C.E. Hayes, S.P. Souza, O.M. Mueller, "The NMR phased array", Magn. Reson. Med., vol.16, pp. 192-225, 1990.

[8] S.M. Wright, L.L. Wald, "Theory and application of array coils in MR spectroscopy", NMR Biomed., vol. 10, pp. 394-410, 1997.

[9] Y. Wang, Z. Shi, Y. Ma, C. Wang, M. Chena nd Y. Chen, 'Supercapacitor Devices Based on Graphene Materials', J. Phys. Chem. C 113, 13103 (2009).

[10] M. Jayalakshmi and K. Balasubramanian, "Review: Simple Capacitors to Supercapacitors," International Journal of Electrochemical Science (2008) 